RIMS-1665

The competition numbers of Johnson graphs

By

Suh-Ryung KIM, Boram PARK and Yoshio SANO

$\underline{\text { March } 2009}$

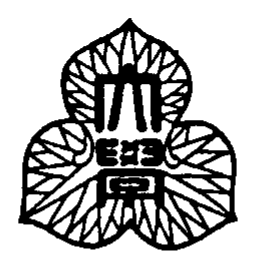

京都大学 数理解析研究所

RESEARCH INSTITUTE FOR MATHEMATICAL SCIENCES

KYOTO UNIVERSITY, Kyoto, Japan 


\title{
The competition numbers of Johnson graphs
}

\author{
SUH-RYUNG KIM *, BORAM PARK \\ Department of Mathematics Education, \\ Seoul National University, Seoul 151-742, Korea. \\ YOSHIO SANO § \\ Research Institute for Mathematical Sciences, \\ Kyoto University, Kyoto 606-8502, Japan.
}

March 2009

\begin{abstract}
The competition graph of a digraph $D$ is a graph which has the same vertex set as $D$ and has an edge between two distinct vertices $x$ and $y$ if and only if there exists a vertex $v$ in $D$ such that $(x, v)$ and $(y, v)$ are arcs of $D$. For any graph $G, G$ together with sufficiently many isolated vertices is the competition graph of some acyclic digraph. The competition number $k(G)$ of a graph $G$ is defined to be the smallest number of such isolated vertices. In general, it is hard to compute the competition number $k(G)$ for a graph $G$ and characterizing a graph by its competition number has been one of important research problems in the study of competition graphs.

The Johnson graph $J(n, d)$ has the vertex set $\left\{v_{X} \mid X \in\left(\begin{array}{c}{[n]} \\ d\end{array}\right)\right\}$, where $\left(\begin{array}{c}{[n]} \\ d\end{array}\right)$ denotes the set of all $d$-subsets of an $n$-set $[n]=\{1, \ldots, n\}$, and two vertices $v_{X_{1}}$ and $v_{X_{2}}$ are adjacent if and only if $\left|X_{1} \cap X_{2}\right|=d-1$. In this paper, we study the edge clique number and the competition number of $J(n, d)$. Especially we give the exact competition numbers of $J(n, 2)$ and $J(n, 3)$.
\end{abstract}

Keywords: competition graph; competition number; edge clique cover; Johnson graph

${ }^{*}$ This work was supported by the Korea Research Foundation Grant funded by the Korean Government (MOEHRD) (KRF-2008-531-C00004).

${ }^{\dagger}$ The author was supported by Seoul Fellowship.

†Corresponding author. E-mail address: kawa22@snu.ac.kr

$\S$ The author was supported by JSPS Research Fellowships for Young Scientists. The author was also supported partly by Global COE program "Fostering Top Leaders in Mathematics". 


\section{Introduction}

The competition graph $C(D)$ of a digraph $D$ is a simple undirected graph which has the same vertex set as $D$ and has an edge between two distinct vertices $x$ and $y$ if and only if there is a vertex $v$ in $D$ such that $(x, v)$ and $(y, v)$ are $\operatorname{arcs}$ of $D$. The notion of a competition graph was introduced by Cohen [3] as a means of determining the smallest dimension of ecological phase space (see also [4]). Since then, various variations have been defi ned and studied by many authors (see [11, 15] for surveys and $[1,2,7,8,9,10$, $12,14,19,20]$ for some recent results). Besides an application to ecology, the concept of competition graph can be applied to a variety of fi elds, as summarized in [17].

Roberts [18] observed that, for a graph $G, G$ together with suffi ciently many isolated vertices is the competition graph of an acyclic digraph. Then he defi ned the competition number $k(G)$ of a graph $G$ to be the smallest number $k$ such that $G$ together with $k$ isolated vertices is the competition graph of an acyclic digraph.

A subset $S$ of the vertex set of a graph $G$ is called a clique of $G$ if the subgraph of $G$ induced by $S$ is a complete graph. For a clique $S$ of a graph $G$ and an edge $e$ of $G$, we say $e$ is covered by $S$ if both of the endpoints of $e$ are contained in $S$. An edge clique cover of a graph $G$ is a family of cliques such that each edge of $G$ is covered by some clique in the family. The edge clique cover number $\theta_{E}(G)$ of a graph $G$ is the minimum size of an edge clique cover of $G$. We call an edge clique cover of $G$ with the minimum size $\theta_{E}(G)$ a minimum edge clique cover of $G$. A vertex clique cover of a graph $G$ is a family of cliques such that each vertex of $G$ is contained in some clique in the family. The vertex clique cover number $\theta_{V}(G)$ of a graph $G$ is the minimum size of a vertex clique cover of $G$. Dutton and Brigham [5] characterized the competition graphs of acyclic digraphs using edge clique covers of graphs.

Roberts [18] observed that the characterization of competition graphs is equivalent to the computation of competition numbers. It does not seem to be easy in general to compute $k(G)$ for a graph $G$, as Opsut [16] showed that the computation of the competition number of a graph is an NP-hard problem (see $[11,13]$ for graphs whose competition numbers are known). For some special graph families, we have explicit formulae for computing competition numbers. For example, if $G$ is a choral graph without isolated vertices then $k(G)=1$, and if $G$ is a nontrivial triangle-free connected graph then $k(G)=|E(G)|-|V(G)|+2$ (see [18]).

In this paper, we study the competition numbers of Johnson graphs. We denote an $n$ set $\{1, \ldots, n\}$ by $[n]$ and the set of all $d$-subsets of an $n$-set by $\left(\begin{array}{c}{[n]} \\ d\end{array}\right)$. The Johnson graph $J(n, d)$ has the vertex set $\left\{v_{X} \mid X \in\left(\begin{array}{c}{[n]} \\ d\end{array}\right)\right\}$, and two vertices $v_{X_{1}}$ and $v_{X_{2}}$ are adjacent if and only if $\left|X_{1} \cap X_{2}\right|=d-1$ (for reference, see [6]). For example, the Johnson graph $J(5,2)$ is given in Figure 1 . As it is known that $J(n, d) \cong J(n, n-d)$, we assume that $n \geq 2 d$. Our main results are the following. 


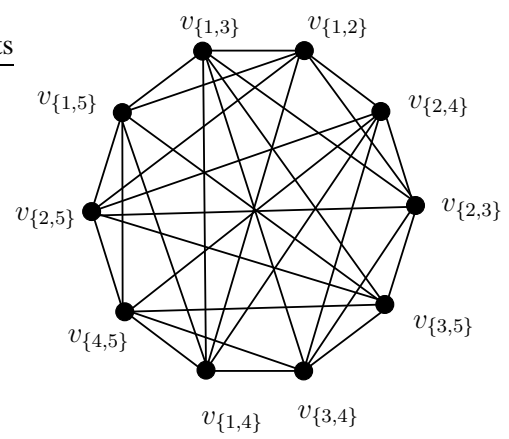

Figure 1: The Johnson graph $J(5,2)$

Theorem 1. For $n \geq 4$, we have $k(J(n, 2))=2$.

Theorem 2. For $n \geq 6$, we have $k(J(n, 3))=4$.

We use the following notation and terminology in this paper. For a digraph $D$, an ordering $v_{1}, v_{2}, \ldots, v_{n}$ of the vertices of $D$ is called an acyclic ordering of $D$ if $\left(v_{i}, v_{j}\right) \in$ $A(D)$ implies $i<j$. It is well-known that a digraph $D$ is acyclic if and only if there exists an acyclic ordering of $D$. For a digraph $D$ and a vertex $v$ of $D$, the out-neighborhood of $v$ in $D$ is the set $\{w \in V(D) \mid(v, w) \in A(D)\}$. A vertex in the out-neighborhood of a vertex $v$ in a digraph $D$ is called a prey of $v$ in $D$. For simplicity, we denote the out-neighborhood of a vertex $v$ in a digraph $D$ by $P_{D}(v)$ instead of usual notation $N_{D}^{+}(v)$. For a graph $G$ and a vertex $v$ of $G$, we defi ne the (open) neighborhood $N_{G}(v)$ of $v$ in $G$ to be the set $\{u \in V(G) \mid u v \in E(G)\}$. We sometimes also use $N_{G}(v)$ to stand for the subgraph induced by its vertices.

\section{A lower bound for the competition number of $J(n, d)$}

In this section, we give lower bounds for the competition number of the Johnson graph $J(n, d)$.

Lemma 3. Let $n$ and $d$ be positive integers with $n \geq 2 d$. For any vertex $x$ of the Johnson graph $J(n, d)$, we have $\theta_{V}\left(N_{J(n, d)}(x)\right)=d$.

Proof. If $d=1$, then $J(n, d)$ is a complete graph and the lemma is trivially true. Assume that $d \geq 2$. Take any vertex $x$ in $J(n, d)$. Then $x=v_{A}$ for some $A \in\left(\begin{array}{c}{[n]} \\ d\end{array}\right)$. For any vertex $v_{A}$ in $J(n, d)$, the set

$$
S_{i}\left(v_{A}\right):=\left\{v_{B} \mid B=(A \backslash\{i\}) \cup\{j\} \text { for some } j \in[n] \backslash A\right\}
$$

forms a clique of $J(n, d)$ for each $i \in A$. To see why, take two distinct vertices $v_{B}$ and $v_{C}$ in $S_{i}\left(v_{A}\right)$. Then $B=(A \backslash\{i\}) \cup\{j\}$ and $C=(A \backslash\{i\}) \cup\{k\}$ for some distinct $j, k \in[n] \backslash A$. Clearly $|B \cap C|=d-1$, and so $v_{B}$ and $v_{C}$ are adjacent in $J(n, d)$. 
Take a vertex $v_{B}$ in $N_{J(n, d)}\left(v_{A}\right)$. Then $B=(A \backslash\{i\}) \cup\{j\}$ for some $i \in A$ and $j \in[n] \backslash A$ and so $v_{B} \in S_{i}\left(v_{A}\right)$. Thus $\left\{S_{i}\left(v_{A}\right) \mid i=1, \ldots, d\right\}$ is a vertex clique cover of $N_{J(n, d)}\left(v_{A}\right)$. Thus $\theta_{V}\left(N_{J(n, d)}\left(v_{A}\right)\right) \leq d$. On the other hand,

$$
\left|((A \backslash\{i\}) \cup\{j\}) \cap\left(\left(A \backslash\left\{i^{\prime}\right\}\right) \cup\left\{j^{\prime}\right\}\right)\right|=d-2
$$

if $i, i^{\prime} \in A$ and $j, j^{\prime} \in[n] \backslash A$ satisfy $i \neq i^{\prime}$ and $j \neq j^{\prime}$ (such $i, i^{\prime}, j, j^{\prime}$ exist since $n \geq 2 d \geq 4)$. This implies that $\theta_{V}\left(N_{J(n, d)}\left(v_{A}\right)\right) \geq d$. Hence $\theta_{V}\left(N_{J(n, d)}\left(v_{A}\right)\right)=d$.

Opsut [16] gave a lower bound for the competition number of a graph $G$ as follows:

$$
k(G) \geq \min \left\{\theta_{V}\left(N_{G}(v)\right) \mid v \in V(G)\right\} .
$$

Together with Lemma 3, we have $k(J(n, d)) \geq d$ for positive integers $n$ and $d$ satisfying $n \geq 2 d$. The following theorem gives a better lower bound for $k(J(n, d))$ if $d \geq 2$.

Theorem 4. For $n \geq 2 d \geq 4$, we have $k(J(n, d)) \geq 2 d-2$.

Proof. Put $k:=k(J(n, d))$. Then there exists an acyclic digraph $D$ such that $C(D)=$ $J(n, d) \cup I_{k}$, where $I_{k}=\left\{z_{1}, z_{2}, \ldots, z_{k}\right\}$ is a set of isolated vertices. Let $x_{1}, x_{2}, \ldots, x_{\left(\begin{array}{c}n \\ d\end{array}\right)}$, $z_{1}, z_{2}, \ldots, z_{k}$ be an acyclic ordering of $D$. Let $v_{1}:=x_{\left(\begin{array}{l}n \\ d\end{array}\right)}$ and $v_{2}:=x_{\left(\begin{array}{l}n \\ d\end{array}\right)-1}$. By Lemma 3 , we have $\theta_{V}\left(N_{J(n, d)}\left(x_{i}\right)\right)=d$ for $i=1, \ldots,\left(\begin{array}{l}n \\ d\end{array}\right)$. Thus $v_{i}$ has at least $d$ distinct prey in $D$, that is,

$$
\left|P_{D}\left(v_{i}\right)\right| \geq d .
$$

Since $x_{1}, x_{2}, \ldots, x_{\left(\begin{array}{c}n \\ d\end{array}\right)}, z_{1}, z_{2}, \ldots, z_{k}$ is an acyclic ordering of $D$, we have

$$
P_{D}\left(v_{1}\right) \cup P_{D}\left(v_{2}\right) \subset I_{k} \cup\left\{v_{1}\right\}
$$

Moreover, we may claim the following:

Claim. For any two adjacent vertices $v_{X_{1}}$ and $v_{X_{2}}$ of $J(n, d)$, we have $\left|P_{D}\left(v_{X_{1}}\right)\right|$ $P_{D}\left(v_{X_{2}}\right) \mid \geq d-1$.

Proof of Claim. Suppose that $v_{X_{1}}$ and $v_{X_{2}}$ are adjacent in $J(n, d)$. Then $\left|X_{1} \cap X_{2}\right|=d-1$ and

$$
\left|[n] \backslash\left(X_{1} \cup X_{2}\right)\right| \geq 2 d-\left|X_{1}\right|-\left|X_{2}\right|+\left|X_{1} \cap X_{2}\right|=d-1 .
$$

We take $d-1$ elements from $[n] \backslash\left(X_{1} \cup X_{2}\right)$, say $z_{1}, z_{2}, \ldots, z_{d-1}$, and put $X_{1} \cap X_{2}:=$ $\left\{y_{1}, y_{2}, \ldots, y_{d-1}\right\}$.

For each $1 \leq j \leq d-1$, we put $Z_{j}:=X_{1} \cup\left\{z_{j}\right\} \backslash\left\{y_{j}\right\}$. Then $\left|Z_{j}\right|=d$ and so $v_{Z_{j}}$ is a vertex in $J(n, d)$. Note that $\left|Z_{j} \cap X_{1}\right|=d-1$ and $\left|Z_{j} \cap X_{2}\right|=d-2$. Thus $v_{Z_{j}}$ is adjacent to $v_{X_{1}}$ while it is not adjacent to $v_{X_{2}}$. Therefore

$$
P_{D}\left(v_{X_{1}}\right) \cap P_{D}\left(v_{Z_{j}}\right) \neq \emptyset \quad \text { and } \quad P_{D}\left(v_{X_{2}}\right) \cap P_{D}\left(v_{Z_{j}}\right)=\emptyset .
$$


Now we show that

$$
P_{D}\left(v_{X_{1}}\right) \backslash P_{D}\left(v_{X_{2}}\right) \supseteq \bigcup_{j=1}^{d-1}\left(P_{D}\left(v_{X_{1}}\right) \cap P_{D}\left(v_{Z_{j}}\right)\right) .
$$

Take a vertex $x$ in $\bigcup_{i=1}^{d-1}\left(P_{D}\left(v_{X_{1}}\right) \cap P_{D}\left(v_{Z_{j}}\right)\right)$. Then $x \in P_{D}\left(v_{X_{1}}\right)$ and $x \in P_{D}\left(v_{Z_{j}}\right)$ for some $j \in\{1, \ldots d-1\}$. Since $P_{D}\left(v_{X_{2}}\right) \cap P_{D}\left(v_{Z_{j}}\right)=\emptyset, x \notin P_{D}\left(v_{X_{2}}\right)$ and so $x \in P_{D}\left(v_{X_{1}}\right) \backslash P_{D}\left(v_{X_{2}}\right)$. Thus (2.3) follows.

Note that for any $j \in\{1, \ldots d-1\}$, since $P_{D}\left(v_{X_{1}}\right) \cap P_{D}\left(v_{Z_{j}}\right) \neq \emptyset$,

$$
\left|P_{D}\left(v_{X_{1}}\right) \cap P_{D}\left(v_{Z_{j}}\right)\right| \geq 1
$$

Moreover, $P_{D}\left(v_{X_{1}}\right) \cap P_{D}\left(v_{Z_{i}}\right)$ and $P_{D}\left(v_{X_{1}}\right) \cap P_{D}\left(v_{Z_{j}}\right)$ are mutually disjoint for $i \neq j$. To see why, note that $\left|Z_{j} \cap Z_{i}\right|=d-2$ for $i \neq j$. Therefore $v_{Z_{i}}$ and $v_{Z_{j}}$ are not adjacent and so $P_{D}\left(v_{Z_{i}}\right) \cap P_{D}\left(v_{Z_{j}}\right)=\emptyset$. Thus

$$
\left(P_{D}\left(v_{X_{1}}\right) \cap P_{D}\left(v_{Z_{i}}\right)\right) \cap\left(P_{D}\left(v_{X_{1}}\right) \cap P_{D}\left(v_{Z_{j}}\right)\right)=\emptyset .
$$

From (2.3), (2.4), and (2.5), it follows that

$$
\left|P_{D}\left(v_{X_{1}}\right) \backslash P_{D}\left(v_{X_{2}}\right)\right| \geq \sum_{i=1}^{d-1}\left|P_{D}\left(v_{X_{1}}\right) \cap P_{D}\left(v_{Z_{j}}\right)\right| \geq d-1 .
$$

This completes the proof of the claim.

Now suppose that $v_{1}$ and $v_{2}$ are not adjacent in $J(n, d)$. Then $v_{1}$ and $v_{2}$ do not have a common prey in $D$, that is,

$$
P_{D}\left(v_{1}\right) \cap P_{D}\left(v_{2}\right)=\emptyset .
$$

By (2.1), (2.2) and (2.6), we have

$$
k+1 \geq\left|P_{D}\left(v_{1}\right) \cup P_{D}\left(v_{2}\right)\right|=\left|P_{D}\left(v_{1}\right)\right|+\left|P_{D}\left(v_{2}\right)\right| \geq 2 d .
$$

Hence $k \geq 2 d-1>2 d-2$.

Next suppose that $v_{1}$ and $v_{2}$ are adjacent in $J(n, d)$. Then $v_{1}$ and $v_{2}$ have at least one common prey in $D$, that is,

$$
\left|P_{D}\left(v_{1}\right) \cap P_{D}\left(v_{2}\right)\right| \geq 1
$$

By the above claim,

$$
\left|P_{D}\left(v_{1}\right) \backslash P_{D}\left(v_{2}\right)\right| \geq d-1 \quad \text { and } \quad\left|P_{D}\left(v_{2}\right) \backslash P_{D}\left(v_{1}\right)\right| \geq d-1 .
$$

Then

$$
\begin{aligned}
k+1 & \geq\left|P_{D}\left(v_{1}\right) \cup P_{D}\left(v_{2}\right)\right| \quad(\text { by }(2.2)) \\
& =\left|P_{D}\left(v_{1}\right) \backslash P_{D}\left(v_{2}\right)\right|+\left|P_{D}\left(v_{2}\right) \backslash P_{D}\left(v_{1}\right)\right|+\left|P_{D}\left(v_{1}\right) \cap P_{D}\left(v_{2}\right)\right| \\
& \geq(d-1)+(d-1)+1 \quad(\text { by }(2.7) \text { and }(2.8)) \\
& =2 d-1 .
\end{aligned}
$$

Hence it holds that $k \geq 2 d-2$. 


\section{An edge clique cover of $J(n, d)$}

In this section, we build a minimum edge clique cover of $J(n, d)$.

Given a Johnson graph $J(n, d)$, we defi ne a family $\mathcal{F}_{d}^{n}$ of cliques of $J(n, d)$ as follows. For each $Y \in\left(\begin{array}{l}{[n]} \\ d-1\end{array}\right)$, we put

$$
S_{Y}:=\left\{v_{X} \mid X=Y \cup\{j\} \text { for } j \in[n]-Y\right\}
$$

Note that $S_{Y}$ is a clique of $J(n, d)$ with size $n-d+1$. We let

$$
\mathcal{F}_{d}^{n}:=\left\{S_{Y} \mid Y \in\left(\begin{array}{c}
{[n]} \\
d-1
\end{array}\right)\right\} .
$$

Then it is not diffi cult to show that $\mathcal{F}_{d}^{n}$ is the collection of cliques of maximum size. Moreover the family $\mathcal{F}_{d}^{n}$ is an edge clique cover of $J(n, d)$. To wee why, take any edge $v_{X_{1}} v_{X_{2}}$ of $J(n, d)$. Then $\left|X_{1} \cap X_{2}\right|=d-1$ and both of $v_{X_{1}}$ and $v_{X_{2}}$ belong to the clique $S_{X_{1} \cap X_{2}} \in \mathcal{F}_{d}^{n}$. Thus $\mathcal{F}_{d}^{n}$ is an edge clique cover of $J(n, d)$.

We will show that $\mathcal{F}_{d}^{n}$ is a minimum edge clique cover of $J(n, d)$. Prior to that, we present the following theorem. For two distinct cliques $S$ and $S^{\prime}$ of a graph $G$, we say $S$ and $S^{\prime}$ are edge disjoint if $\left|S \cap S^{\prime}\right| \leq 1$.

Theorem 5. $\theta_{E}(J(n, d))=\left(\begin{array}{c}n \\ d-1\end{array}\right)$ and any minimum edge clique cover of $J(n, d)$ consists of edge disjoint maximum cliques.

Proof. Let $\mathcal{E}$ be a minimum edge clique cover for $J(n, d)$, that is, $\theta_{E}(J(n, d))=|\mathcal{E}|$. Since $\mathcal{F}_{d}^{n}$ is an edge clique cover with $\left|\mathcal{F}_{d}^{n}\right|=\left(\begin{array}{c}n \\ d-1\end{array}\right)$, we have $\theta_{E}(J(n, d)) \leq\left(\begin{array}{c}n \\ d-1\end{array}\right)$.

Now we show that $|\mathcal{E}| \geq\left(\begin{array}{c}n \\ d-1\end{array}\right)$. Since the size of a maximum clique is $n-d+1$, we have $|E(S)| \leq\left(\begin{array}{c}n-d+1 \\ 2\end{array}\right)$ for each $S \in \mathcal{E}$ where $E(S)=\left(\begin{array}{c}S \\ 2\end{array}\right)$. Therefore,

$$
|E(J(n, d))| \leq \sum_{S \in \mathcal{E}}|E(S)| \leq\left(\begin{array}{c}
n-d+1 \\
2
\end{array}\right) \times|\mathcal{E}|,
$$

and the fi rst equality holds if and only if none of two distinct cliques in $\mathcal{E}$ have a common edge, and the second equality holds if and only if any element of $\mathcal{E}$ is a maximum clique in $J(n, d)$.

Since the Johnson graph $J(n, d)$ is a $d(n-d)$-regular graph and the number of vertices of $J(n, d)$ is $\left(\begin{array}{l}n \\ d\end{array}\right)$,

$$
|E(J(n, d))|=\frac{1}{2} d(n-d) \times\left(\begin{array}{l}
n \\
d
\end{array}\right)=\left(\begin{array}{c}
n-d+1 \\
2
\end{array}\right) \times\left(\begin{array}{c}
n \\
d-1
\end{array}\right) .
$$

From (3.2) and (3.3), it follows that $\left(\begin{array}{c}n-d+1 \\ 2\end{array}\right) \times\left(\begin{array}{c}n \\ d-1\end{array}\right) \leq\left(\begin{array}{c}n-d+1 \\ 2\end{array}\right) \times|\mathcal{E}|$. Thus we have $\left(\begin{array}{c}n \\ d-1\end{array}\right) \leq|\mathcal{E}|$. Hence we can conclude that $\theta_{E}(J(n, d))=\left(\begin{array}{c}n \\ d-1\end{array}\right)$.

Furthermore, two equalities in (3.2) must hold, and therefore any minimum edge clique cover of $J(n, d)$ consists of edge disjoint maximum cliques. 

5:

Since $\left|\mathcal{F}_{d}^{n}\right|=\left(\begin{array}{c}n \\ d-1\end{array}\right)$, the following corollary is an immediate consequence of Theorem

Corollary 6. The edge clique cover $\mathcal{F}_{d}^{n}$ of $J(n, d)$ defined in (3.1) is a minimum edge clique cover of $J(n, d)$.

\section{Proofs of Theorems 1 and 2}

First, we defi ne an order $\prec$ on the set $\left(\begin{array}{c}{[n]} \\ d\end{array}\right)$ as follows. Take two distinct elements $X_{1}$ and $X_{2}$ in $\left(\begin{array}{c}{[n]} \\ d\end{array}\right)$. Let $X_{1}=\left\{i_{1}, i_{2}, \ldots, i_{d}\right\}$ and $X_{2}=\left\{j_{1}, j_{2}, \ldots, j_{d}\right\}$ where $i_{1}<\ldots<i_{d}$ and $j_{1}<\ldots<j_{d}$. Then we defi ne $X_{1} \prec X_{2}$ if there exists $t \in\{1, \ldots, d\}$ such that $i_{s}=j_{s}$ for $1 \leq s \leq t-1$ and $i_{t}<j_{t}$. It is easy to see that $\prec$ is a total order.

Now we prove Theorem 1 .

Proof of Theorem 1. As $k(J(n, 2)) \geq 2$ by Theorem 4, it remains to show $k(J(n, 2)) \leq$ 2. We defi ne a digraph $D$ as follows:

$$
V(D)=V(J(n, 2)) \cup I_{2}
$$

where $I_{2}=\left\{z_{1}, z_{2}\right\}$, and

$$
A(D)=\bigcup_{i=1}^{n-2}\left\{\left(x, v_{\{i+1, i+2\}}\right) \mid x \in S_{\{i\}} \in \mathcal{F}_{2}^{n}\right\} \cup \bigcup_{i=1}^{2}\left\{\left(x, z_{i}\right) \mid x \in S_{\{n-2+i\}} \in \mathcal{F}_{2}^{n}\right\} .
$$

Since the vertices of each clique in the edge clique cover $\mathcal{F}_{2}^{n}$ has a common prey in $D$, it holds that $C(D)=J(n, 2) \cup I_{2}$. Each vertex in $S_{i}$ is denoted by $v_{X}$ for some $X \in\left(\begin{array}{c}{[n]} \\ 2\end{array}\right)$ which contains $i$. Then by the defi nition of $\prec, v_{X} \prec v_{\{i+1, i+2\}}$ for $i=1, \ldots, n-2$. Thus, there exists an arc from a vertex $x$ to a vertex $y$ in $D$ if and only if either $x=v_{X}$ and $y=v_{Y}$ with $X \prec Y$, or $x=v_{X}$ and $y=z_{i}$ with $X \in S_{\{n-1\}} \cup S_{\{n\}}$ and $i \in\{1,2\}$. Therefore $D$ is acyclic. Thus we have $k(J(n, 2)) \leq 2$ and this completes the proof.

Proof of Theorem 2. By Theorem 4, we have $k(J(n, 3)) \geq 4$. It remains to show $k(J(n, 3)) \leq$ 4. We defi ne a digraph $D$ as follows:

$$
V(D)=V(J(n, 3)) \cup I_{4}
$$


where $I_{4}=\left\{z_{1}, z_{2}, z_{3}, z_{4}\right\}$, and

$$
\begin{aligned}
A(D)= & \bigcup_{i=1}^{n-3} \bigcup_{j=i+1}^{n-2}\left\{\left(x, v_{\{i, j+1, j+2\}}\right) \mid x \in S_{\{i, j\}} \in \mathcal{F}_{3}^{n}\right\} \\
& \cup \bigcup_{i=1}^{n-3}\left\{\left(x, v_{\{i+1, i+2, i+3\}}\right) \mid x \in S_{\{i, n-1\}} \in \mathcal{F}_{3}^{n}\right\} \\
& \cup \bigcup_{i=1}^{n-4}\left\{\left(x, v_{\{i+1, i+2, i+4\}}\right) \mid x \in S_{\{i, n\}} \in \mathcal{F}_{3}^{n}\right\} \\
& \cup \bigcup_{i=1}^{3}\left\{\left(x, z_{i}\right) \mid x \in S_{\{n-4+i, n\}} \in \mathcal{F}_{3}^{n}\right\} \\
& \cup\left\{\left(x, z_{4}\right) \mid x \in S_{\{n-2, n-1\}} \in \mathcal{F}_{3}^{n}\right\} .
\end{aligned}
$$

It is easy to check that

$$
\begin{aligned}
\mathcal{F}_{3}^{n}= & \left\{S_{\{i, j\}} \mid i=1, \ldots, n-3 ; j=i+1, \ldots, n-2\right\} \cup\left\{S_{\{i, n-1\}} \mid i=1, \ldots, n-3\right\} \\
& \cup\left\{S_{\{i, n\}} \mid i=1, \ldots, n-4\right\} \cup\left\{S_{\{n-3, n\}}, S_{\{n-2, n\}}, S_{\{n-1, n\}}\right\} \cup\left\{S_{\{n-2, n-1\}}\right\} .
\end{aligned}
$$

Thus $C(D)=J(n, 3) \cup I_{4}$. Moreover, any vertex $x \in S_{\{i, j\}}$ is denoted by $v_{X}$ for some $X \in\left(\begin{array}{c}{[n]} \\ 3\end{array}\right)$ which contains $i$ and $j$. By the defi nition of $\prec, X \prec\{i, j+1, j+2\}$. In a similar manner, for $x$ in other cliques in $\mathcal{F}_{3}^{n}$, we may show that $(x, y) \in A(D)$ if and only if either $x=v_{X}$ and $y=v_{Y}$ with $X \prec Y$, or $x=v_{X}$ and $y=z_{i}$ with $X \in S_{\{n-3, n\}} \cup S_{\{n-2, n\}} \cup S_{\{n-1, n\}} \cup S_{\{n-2, n-1\}}$ and $i \in\{1,2,3,4\}$. Thus $D$ is acyclic. Hence $k(J(n, 3)) \leq 4$.

\section{Concluding Remarks}

In this paper, we gave some lower bounds for the competition numbers of Johnson graphs, and computed the competition numbers of Johnson graphs $J(n, 2)$ and $J(n, 3)$. It would be natural to ask: What is the exact value of the competition number of a Johnson graph $J(n, 4)$ for $n \geq 8$ ? Eventually, what is the exact values of the competition numbers of Johnson graphs $J(n, q)$ ?

\section{References}

[1] H. H. Cho and S. -R. Kim: The competition number of a graph having exactly one hole, Discrete Math. 303 (2005) 32-41.

[2] H. H. Cho, S. -R. Kim and Y. Nam: On the trees whose 2-step competition numbers are two, Ars Combin. 77 (2005) 129-142. 
[3] J. E. Cohen: Interval graphs and food webs: a fi nding and a problem, Document 17696-PR, RAND Corporation, Santa Monica, CA (1968).

[4] J. E. Cohen: Food webs and Niche space, Princeton University Press, Princeton, NJ (1978).

[5] R. D. Dutton and R. C. Brigham: A characterization of competition graphs, Discrete Appl. Math. 6 (1983) 315-317.

[6] C. Godsil and G. Royle: Algebraic Graph Theory, Graduate Texts in Mathematics 207, Springer-Verlag (2001).

[7] S. G. Hartke: The elimination procedure for the phylogeny number, Ars Combin. 75 (2005) 297-311.

[8] S. G. Hartke: The elimination procedure for the competition number is not optimal, Discrete Appl. Math. 154 (2006) 1633-1639.

[9] G. T. Helleloid: Connected triangle-free $m$-step competition graphs, Discrete Appl. Math. 145 (2005) 376-383.

[10] W. Ho: The $m$-step, same-step, and any-step competition graphs, Discrete Appl. Math. 152 (2005) 159-175.

[11] S. -R. Kim: The competition number and its variants, Quo Vadis, Graph Theory, (J. Gimbel, J. W. Kennedy, and L. V. Quintas, eds.), Annals of Discrete Mathematics 55, North-Holland, Amsterdam (1993) 313-326.

[12] S. -R. Kim: Graphs with one hole and competition number one, J. Korean Math. Soc. 42 (2005) 1251-1264.

[13] S. -R. Kim and F. S. Roberts: Competition numbers of graphs with a small number of triangles, Discrete Appl. Math. 78 (1997) 153-162.

[14] S. -R. Kim and Y. Sano: The competition numbers of complete tripartite graphs, Discrete Appl. Math. 156 (2008) 3522-3524.

[15] J. R. Lundgren: Food Webs, Competition Graphs, Competition-Common Enemy Graphs, and Niche Graphs, in Applications of Combinatorics and Graph Theory to the Biological and Social Sciences, IMH Volumes in Mathematics and Its Application 17 Springer-Verlag, New York, (1989) 221-243.

[16] R. J. Opsut: On the computation of the competition number of a graph, SIAM J. Algebraic Discrete Methods 3 (1982) 420-428. 
[17] A. Raychaudhuri and F. S. Roberts: Generalized competition graphs and their applications, Methods of Operations Research, 49 Anton Hain, Königstein, West Germany, (1985) 295-311.

[18] F. S. Roberts: Food webs, competition graphs, and the boxicity of ecological phase space, Theory and applications of graphs (Proc. Internat. Conf., Western Mich. Univ., Kalamazoo, Mich., 1976) (1978) 477-490.

[19] F. S. Roberts and L. Sheng: Phylogeny numbers for graphs with two triangles, Discrete Appl. Math. 103 (2000) 191-207.

[20] M. Sonntag and H. -M. Teichert: Competition hypergraphs, Discrete Appl. Math. 143 (2004) 324-329. 